\title{
Analysis of Greater Tuberosity from the Center of the Humeral Head: Progression to Femoralization
}

\author{
Jun-Seok Lee, Hyun Seok Song ${ }^{\square}$, Hyungsuk Kim, Hyung Moon Yoon, Sung Bin Han \\ Department of Orthopedic Surgery, Eunpyeong St. Mary's Hospital, College of Medicine, The Catholic University of Korea, Seoul, Korea
}

Background: Progression of the tear size and erosion of the greater tuberosity (femoralization) in the supraspinatus tear makes it difficult to repair or increases the risk of a re-tear. This study examined the proximal articular surface and greater tuberosity of the humeral head in plain radiography.

Methods: Two-hundred forty-seven cases, whose anteroposterior (AP) radiographs were taken correctly, were included from 288 cases, in whom the status of the supraspinatus had been confirmed by surgery. After downloading the plain AP radiograph as DICOM, the radius of the circle apposed at the superior half of the articular surface of the head, and the distance between the circle and the farthest point of the greater tuberosity ('height' of the greater tuberosity) were calculated using the software (TechHime, Korea). MRI checked the number of torn tendons and degree of muscular atrophy.

Results: The following were encountered: 93 intact supraspinatus, 50 partial-thickness tears, and 104 full-thickness tears. In the analysis using the 93 intact cases, the average radius of the rotation center was $25.3 \mathrm{~mm}$ in male and $22.3 \mathrm{~mm}$ in female. The average height of the greater tuberosity from the circle with the same rotation center was $4.3 \mathrm{~mm}$ in male and $4.2 \mathrm{~mm}$ in female with no statistical significance. The correlation between the reparability of supraspinatus and height of the greater tuberosity, fatty infiltration, and muscular atrophy was confirmed.

Conclusions: The height of the greater tuberosity from the circle with the same rotation center was $4.3 \mathrm{~mm}$ in male and $4.2 \mathrm{~mm}$ in female. This height was strongly correlated with muscular atrophy and fatty infiltration of the supraspinatus tendon.

(Clin Shoulder Elbow 2019;22(4):216-219)

Key Words: Supraspinatus tear; Height; Grater tuberosity; Femoralization; Muscular Atrophy

\section{Introduction}

The size of rotator cuff tears progresses with time. ${ }^{1-3)}$ In the case of chronic and larger tears, there is difficulty in repair as a result of muscular atrophy ${ }^{4)}$ and fatty infiltration. ${ }^{5,6)}$ Massive rotator cuff tears are defined as tears involving more than two cuff tendons ${ }^{1)}$ or tears more than $5 \mathrm{~cm}^{7)}$ In these cases, the re-tear rate is high.

A decrease in the acromio-humeral distance $(\mathrm{AHD})^{8)}$ in the plain radiographs is a clue of the irreparability of the torn cuff. According to many research reports thus far, a higher degree of fatty degeneration in the rotator cuff in computed tomography (CT) or magnetic resonance imaging (MRI) indicates poorer clinical findings, even after surgery. In plain radiography, there are clues, such as the anterolateral bony spur of acromion, osteophyte formation of the greater tuberosity, and a decrease in AHD.

Therefore, the authors aimed to identify the changes in the greater tuberosity and determine the relevance between fatty infiltration in MRI and the height from the center of rotation to the greater tuberosity.

Received November 22, 2019. Revised November 25, 2019. Accepted November 26, 2019.

Correspondence to: Hyun Seok Song

Department of Orthopedic Surgery, Eunpyeong St. Mary's Hospital, College of Medicine, The Catholic University of Korea, 1021 Tongil-ro, Eunpyeong-gu, Seoul 03312, Korea

Tel: +82-2-2030-4628, Fax: +82-2-2030-4629, E-mail: hssongmd@hanmail.net, ORCID: https://orcid.org/0000-0002-7844-2293

IRB approval: The Catholic University of Korea, St. Paul's Hospital (No. PC10OISI0026).

Financial support: None. Conflict of interests: None. 


\section{Methods}

The study protocol was approved by the Institutional Review Board, which waived the requirement for informed consent due to the retrospective nature of this study. At one university hospital, 288 supraspinatus tendon tears were enrolled. Among them, 41 cases were excluded due to inappropriate plain anteroposterior (AP) radiographs; 247 cases were enrolled.

\section{Measurement of Radius and Greater Tuberosity of Humeral Head}

A simple AP shoulder radiograph was saved in the form of DICOM (Digital Imaging and Communications in Medicine). The software program (ViewRex; TechHime, Seoul, Korea) used in this study, was developed as a tool menu for this study. This software can make a circle on a plain radiograph, change the radius, and move the location. By adjusting the radius and location of the circle, the circle could be matched to the articular surface. At this location, it was programmed for an automatic calculation of the radius of a circle and the difference between the radius and highest greater tuberosity. The difference between the radius of a circle and the highest point of greater tuberosity was called the 'height' of the greater tuberosity from the center of rotation (Fig. 1).

\section{Reparability of the Supraspinatus Tendon}

Videos taken during surgery and medical records were analyzed retrospectively, and the number of torn rotator cuffs and the degree of reparability were analyzed. The cases were allocated into two groups. One group contained cases with a torn edge that was pulled easily to the original footprint. Another

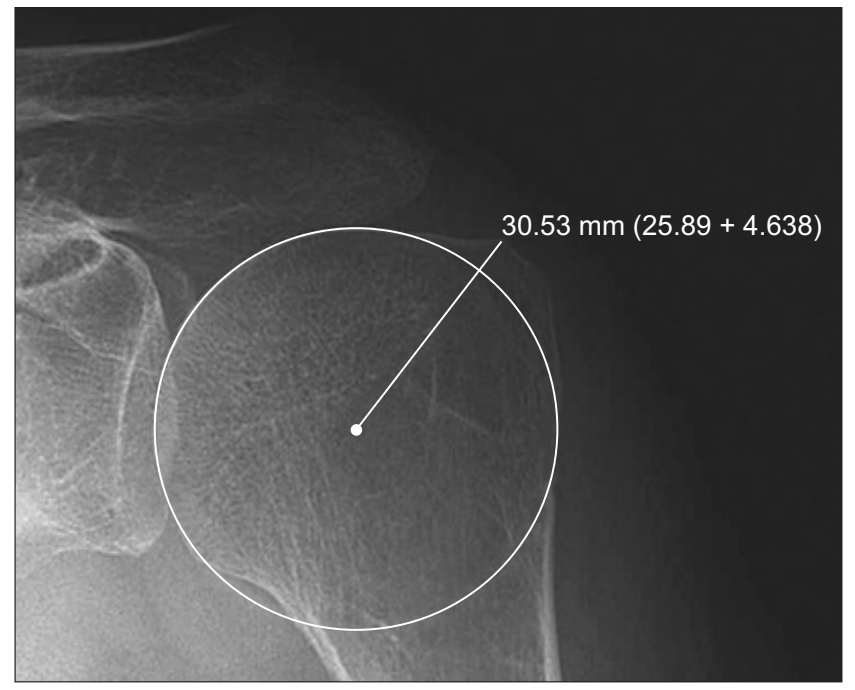

Fig. 1. Plain radiograph captured measuring the rotation center of the humeral head and height of the greater tuberosity (distance from the circle to the farthest point). group was cases, in whom the interval slide ${ }^{10)}$ needed to be mobilized or the partial repair was done because it was not pulled to the original footprint despite the releasing techniques. For the reparability of the supraspinatus tendon, grade 1 and 2 was classified as normal, and grade 2 was classified as a case that is repairable on the originally attached portion easily without any tension in the tendon. Grade 3 was classified as a case that the movement of the tendon was not easy enough to require the anterior or rear-interval slide technique or a case when the state of torn tendon became thinner. Grade 4 was classified as follows: a case that partial repair was possible despite any device; the case with a re-tear was identified during follow up, but the suture was conducted using interval slide technique; or a case that cuff arthropathy was severe enough to perform reverse total shoulder arthroplasty.

\section{Assessment of Atrophy and Fatty Infiltration of the Supraspinatus Tendon}

The degree of fatty infiltration of the supraspinatus tendon

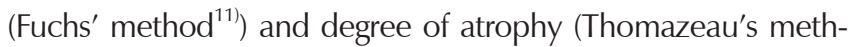
$\mathrm{od}^{12)}$ ) were measured in the preoperative MRI images.

Fuchs et al. ${ }^{11)}$ used CT and MRI to assess the fatty infiltration of a muscle of rotator cuff tear instead of Goutallier classification (five stages). Thomazeau et al. ${ }^{12)}$ used MRI to grade muscle atrophy. This method allowed a reliable measurement of the supraspinatus muscle atrophy by calculating the occupation ratio (R), which is the ratio between the surface of the cross-section of the muscle belly and that of the fossa. The classification was divided into three stages. Stage 1 was normal to slight atrophy of the supraspinatus muscle $(R=1.00$ to 0.60$)$. Stage 2 was moderate atrophy $(R=0.60$ to 0.40$)$. Stage 3 was severe atrophy $(R<0.40){ }^{13)}$

\section{Statistical Analysis}

Statistical analysis SPSS 12.0k (SPSS Inc., Chicago, IL, USA) was used, and the 95\% confidence interval was tested. The correlation in the difference between males and females was analyzed (parametric analysis with Pearson's correlation test; non-parametric analysis with Spearman's correlation test). To measure the difference in the average between males and females, a Student's t-test was used for parametric analysis and a Mann-Whitney test was used for non-parametric analysis.

One-way analysis of variance (ANOVA) was used to measure the degree of reparability in the supraspinatus tendon, the degree of fatty infiltration, and muscular atrophy, highest portion of the greater tuberosity from the center of rotation, and the correlation was analyzed.

\section{Results}

Among the 247 cases, there were 93 intact supraspinatus, 50 partial-thickness tears, and 104 full-thickness tears. 


\section{Measurement of the Radius and Greater Tuberosity of Humeral Head}

For 93 intact supraspinatus cases, the greater tuberosity was analyzed as normal differences. The average of the proximal radius in the humeral head was $25.3 \mathrm{~mm}$ in males and $22.3 \mathrm{~mm}$ in females, which showed a significant difference $(p<0.001)$. The average height from the center of rotation was $4.3 \mathrm{~mm}$ in males and $4.2 \mathrm{~mm}$ in females, but there was no significant difference (Mann-Whitney test, $p=0.795$; Student's t-test, $p=0.438$ ). A relationship between the radius in the humeral head and height from the center of rotation to the greater tuberosity was observed, but the correlation was weak (Pearson correlation coefficient $r=0.157$ : Spearman correlation coefficient $r=0.110$ ).

\section{Assessment of Atrophy and Fatty Infiltration of the Supraspinatus Tendon}

In the grades depending on the reparability of the supraspinatus tendon, there were significant correlations $(r>0.2)$ between the height from the center of rotation, degree of fatty degeneration, degree of atrophy, and reparability grades (Table 1).

\section{Discussion}

The height from the head center, which was analyzed in this study, showed a significant correlation with the fatty infiltration, muscle atrophy in the radiologic images, and reparability during arthroscopic repair.

Currently, there are few reliable options to manage irreparable rotator cuff tears. On the other hand, atrophy in the tendon is severe in cases of massive tears. Thus, the clinical results from the repair of the torn tendon are not good if it is difficult to recover to the originally attached portion with the general method, or if muscle atrophy becomes serious. Therefore, good results can be achieved if conducting appropriate repair after considering the time before the condition of the tendon reaches a serious level.

As special radiological evaluations to identify the condition of rotator cuffs, ultrasonography, arthrography, CT arthrography, and MRI were used, but there might be situations that such special radiologic evaluations cannot be applied owing to cost and social status reasons.

Many methods have been proposed to assess the reparability of rotator cuff tears. The widely used methods are measuring AHD and fatty infiltration. ${ }^{14)}$

Table 1. Correlation Coefficients with the Reparability Grades

\begin{tabular}{lccc}
\hline \multicolumn{1}{c}{ Variable } & $\begin{array}{c}\text { Greater tuberosity } \\
\text { height }\end{array}$ & $\begin{array}{c}\text { Fatty } \\
\text { infiltration }\end{array}$ & $\begin{array}{c}\text { Muscle } \\
\text { atrophy }\end{array}$ \\
\hline Pearson's & -0.439 & 0.766 & 0.707 \\
Spearman's rho & -0.332 & 0.751 & 0.718 \\
\hline
\end{tabular}

Superior migration of the humeral head is a well-known phenomenon in the late stage of rotator cuff disease or massive tears. Therefore, many surgeons use AHD to evaluate the rotator cuff tear and surgical decision-making. On the other hand, it is unknown whether tendon involvement, tear sizes, or muscle degeneration is the most important structural change in the reduced AHD. The methods of measuring AHD among studies vary according to anatomical landmarks. Moreover, the lack of consistent standardized methods to measure the AHD may lead to a lack of specificity and large variance.

Fatty infiltration is another widely used method to assess reparability. ${ }^{15)}$ After rotator cuff tears, atrophy of muscle fibers, ${ }^{16)}$ fibrosis, and accumulation of fat within and around muscle fibers are often observed. This pathophysiological change is referred to as fatty infiltration, which is defined broadly as the loss of skeletal muscle mass and strength that occurs with aging. Fatty infiltration is largely dependent on the size and the chronicity of rotator cuff tears. The degree of these changes increases with increasing tear size and time elapsed from the rupture of the tendon, and often represents the limiting factor for tendon reconstruction. ${ }^{17)}$ Gerber et al. ${ }^{17)}$ found from their study in a retraction muscle after tendon tear that the pennation angle is increased, and is most likely not followed by an increase in the number of muscle fibers but by a more unfavorable mechanical angle of the fibers to the central muscle tendon. This means that the stage of fatty infiltration is not only a concern, but the force and quality of the remaining fibers are also likely to be somewhat underestimated in the more retracted and fatty infiltrated muscle. From the authors' personal experience, in many cases with cuff tears with grade 3 fatty infiltration, it was possible to reduce the torn tendon to the footprint in actual surgery.

As the suggestive findings of rotator cuff disease in plain radiographs, there are anterolateral acromion spur and greater tuberosity osteophyte formation ${ }^{9)}$ as well as a reduction in AHD. Acromion or greater tuberosity spur are seen from the early stages of rotator cuff disease. Even if the osteophyte of the acromion is large, it cannot be associated with the rotator cuff atrophy; there is insufficient evidence to assess the size of a tear as large. Decreased AHD is observed clearly after rotator cuff joint disease occurs, but there are many errors depending on the method used to measure the AHD.

Regarding the findings that the humeral head appears almost circular with the reduced height from the center of rotation to the greater tuberosity, humeral head femoralization is observed with acromial acetabularization in cases in which rotator cuff disease progressed. In many cases, however, a decrease in height from the center of rotation was observed unless there was an acromial acetabularization or serious reduction in AHD. From the phenomenon that there is upward movement of the humeral head during abduction, the long-term findings can be used to determine whether to proceed. ${ }^{18)}$ 
This study analyzed the normal range of height from the rotation center. In the plain radiographs, the distance between the center of rotation, which belongs to the articular surface on the proximal portion of the proximal humeral head and the greater tuberosity (height from the center of rotation to greater tuberosity), was $4 \mathrm{~mm}$, and there was no difference between males and females. In addition, the height showed a good correlation with the reparability of the supraspinatus tears. If the height is reduced significantly, it can be expected that it will be difficult to reduce it to the footprint during surgery.

This study had some limitations in the study. The number of cases analyzed was insufficient. The reparability grades could also have been affected by the authors' surgical skills. ${ }^{19)}$

\section{Conclusion}

The height of the greater tuberosity from the circle with the same rotation center was $4.3 \mathrm{~mm}$ in male and $4.2 \mathrm{~mm}$ in female. This height strongly correlated with muscular atrophy and fatty infiltration of the supraspinatus tendon.

\section{References}

1. Zumstein MA, Jost B, Hempel J, Hodler J, Gerber C. The clinical and structural long-term results of open repair of massive tears of the rotator cuff. J Bone Joint Surg Am. 2008; 90(11):2423-31. doi: 10.2106/JBJS.G.00677.

2. Burkhart SS, Danaceau SM, Pearce CE Jr. Arthroscopic rotator cuff repair: analysis of results by tear size and by repair technique-margin convergence versus direct tendon-tobone repair. Arthroscopy. 2001;17(9):905-12. doi: 10.1053/ jars.2001.26821.

3. Gueniche J, Bierry G. Rotator cuff muscles fatty infiltration increases with age: retrospective review of 210 patients with intact cuff on computed tomography arthrography. J Shoulder Elbow Surg. 2019;28(4):617-24. doi: 10.1016/j.jse. 2018.09.020.

4. Matsumura N, Oguro S, Okuda S, et al. Quantitative assessment of fatty infiltration and muscle volume of the rotator cuff muscles using 3-dimensional 2-point Dixon magnetic resonance imaging. J Shoulder Elbow Surg. 2017;26(10):e309-18. doi: 10.1016/j.jse.2017.03.019.

5. Kuptniratsaikul V, Laohathaimongkol T, Umprai V, Yeekian C, Prasathaporn N. Pre-operative factors correlated with arthroscopic reparability of large-to-massive rotator cuff tears. BMC Musculoskelet Disord. 2019;20(1):111. doi: 10.1186/ s12891-019-2485-4.

6. Valencia AP, Lai JK, lyer SR, et al. Fatty infiltration is a prognostic marker of muscle function after rotator cuff tear. Am J Sports
Med. 2018;46(9):2161-9. doi: 10.1177/0363546518769267.

7. Cofield RH. Rotator cuff disease of the shoulder. J Bone Joint Surg Am. 1985;67(6):974-9.

8. Ellman H, Hanker G, Bayer M. Repair of the rotator cuff. Endresult study of factors influencing reconstruction. J Bone Joint Surg Am. 1986;68(8):1136-44.

9. Cone RO 3rd, Resnick D, Danzig L. Shoulder impingement syndrome: radiographic evaluation. Radiology. 1984;150(1):29-33. doi: 10.1148/radiology.150.1.6689783.

10. Tauro JC. Arthroscopic "interval slide" in the repair of large rotator cuff tears. Arthroscopy. 1999;15(5):527-30. doi: 10.1053/ar.1999.v15.0150521.

11. Fuchs B, Weishaupt D, Zanetti M, Hodler J, Gerber C. Fatty degeneration of the muscles of the rotator cuff: assessment by computed tomography versus magnetic resonance imaging. J Shoulder Elbow Surg. 1999;8(6):599-605. doi: 10.1016/ s1058-2746(99)90097-6.

12. Thomazeau H, Rolland $Y$, Lucas C, Duval JM, Langlais F. Atrophy of the supraspinatus belly. Assessment by MRI in 55 patients with rotator cuff pathology. Acta Orthop Scand. 1996;67(3):264-8. doi: 10.3109/17453679608994685.

13. Jeong JY, Chung PK, Lee SM, Yoo JC. Supraspinatus muscle occupation ratio predicts rotator cuff reparability. J Shoulder Elbow Surg. 2017;26(6):960-6. doi: 10.1016/j.jse.2016.11.001.

14. Dwyer T, Razmjou H, Henry P, Gosselin-Fournier S, Holtby $R$. Association between pre-operative magnetic resonance imaging and reparability of large and massive rotator cuff tears. Knee Surg Sports Traumatol Arthrosc. 2015;23(2):415-22. doi: 10.1007/s00167-013-2745-z.

15. Di Benedetto P, Beltrame A, Cicuto C, et al. Rotator cuff tears reparability index based on pre-operative MRI: our experience. Acta Biomed. 2019;90(1-S):36-46. doi: 10.23750/abm. v90i1-S.8074.

16. Kim JY, Park JS, Rhee YG. Can preoperative magnetic resonance imaging predict the reparability of massive rotator cuff tears? Am J Sports Med. 2017;45(7):1654-63. doi: 10.1177/ 0363546517694160.

17. Gerber C, Schneeberger AG, Hoppeler H, Meyer DC. Correlation of atrophy and fatty infiltration on strength and integrity of rotator cuff repairs: a study in thirteen patients. J Shoulder Elbow Surg. 2007;16(6):691-6. doi: 10.1016/j.jse.2007.02.122.

18. Burkhart SS. Fluoroscopic comparison of kinematic patterns in massive rotator cuff tears. A suspension bridge model. Clin Orthop Relat Res. 1992;(284):144-52. doi: 10.1097/00003086199211000-00018.

19. Gartsman GM. Arthroscopic assessment of rotator cuff tear reparability. Arthroscopy. 1996;12(5):546-9. doi: 10.1016/ s0749-8063(96)90192-9. 